fixed points of light of little known significance. The second phase started in the late 1800 s when astrophotography and spectroscopy spawned the discipline of astrophysics. During this period planetary investigation degenerated into a Cinderella science. The early 1960 s saw the dawn of planetology. Since then spacecraft have been flung round, onto and into all of the planets known to the ancients, producing a veritable explosion of knowledge.

The first book under review is The New Solar System. Very rarely does one come across such an excellent account; I feel honoured to have a first edition as it is bound to run to many printings. The editors have collected together 20 chapters, each of about ten pages, written by a roll call of American scientists which reads like a Who's Who of US planetology. It is invidious to omit names, but as an indication of the standard it includes Eddy on the Sun, Chapman on asteroids, Burns on planetary rings, Wood on meteorites, Masursky on Mars, Shoemaker on the role of fragmentation and so on. The book concentrates on the comparative approach. It is much easier to discuss the surfaces of the terrestrial planets (Mercury, Venus, Earth and Mars) in one chapter and, for example, their atmospheres in another than to have an isolationist attitude to individual planets.

The joy of reading the book is greatly enhanced by the illustrations. Not only does it bulge with space photographs, but it also contains a collection of coloured illustrations by artists Charles Wheeler, Don Davies and Jon Lomberg.

The editor's aim was to provide a book about the third phase of planetary exploration, the space-age planets, a book which would make enjoyable reading for those with either a professional or a casual interest. They have succeeded admirably. The fact that the publishers have produced this large format, beautifully illustrated, quality book at less than $£ 10$ is verging on the miraculous. I advise all readers to buy it.

The second book is Fred Whipple's Orbiting the Sun: Planets and Satellites of the Solar System. This is a completely revised and updated edition of Whipple's. classic text, Earth, Moon and Planets, which was first published in 1941. Whipple is Philips Professor of Astronomy, Emeritus, at Harvard University and Senior Scientist, Emeritus, at the Smithsonian Institution Astrophysical Observatory. He has devoted his life to the study of astronomy and space science, especially meteors, comets, space exploration planning, satetlite tracking, novae and supernovae, Solar System evolution and practical astronomy; it is fascinating to read the view of such a man about the place of Earth and Moon within the planetary context. Whipple writes for the general reader and mathematics are avoided; however, the book does present the basic foundation of planetary astronomy and

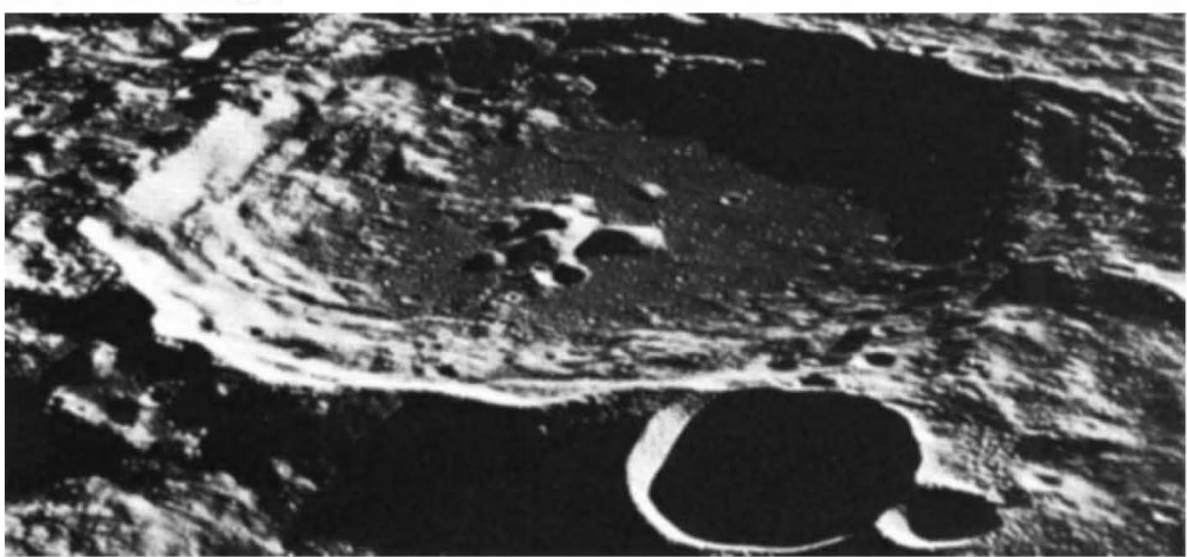

The crater Daedalus on the far side of the Moon, photographed by Armstrong and Aldrin on July 21 , 1969. The picture is taken from The Atlas of the Universe by Patrick Moore, a new edition of which has just been published by Mitchell Beazley, price $£ 19.95$.

does stress the underlying scientific principles. The author has also managed to integrate the three phases of Solar System investigation and we realize that all still have a role to play. We might move from bleary telescope images to the eye-opening, crisp detail of the space probe, but the Earth-based telescopes have recently revealed Pluto's moon, the rings of Uranus and Apollo asteroids. Also we realize that stars and planets are not to be compartmentalized. Whipple leaves us in no doubt that the origins and deaths of stars are intricately connected with the origin and evolution of our planetary system. The book is well written, well illustrated, well worth reading and a worthy successor to the previous editions.

My final book is Daytime Star: the Story of Our Sun by Simon Mitton. The word "Story" in the title describes the book well - Mitton dives into the subject and returns with a net bulging with intrigues. Why did ancient man worship the Sun and build stone circles to track its path? What drives the sunspots? Does the solar energy output vary slightly and thus trigger ice ages? What do neutrinos tell us about the solar interior? What have we learnt from space about the solar atmosphere? Mitton has a racy style and we find atomic particles sloshing, neutrons bashing, gases toasting and gravity crunching.

There is much to be learnt about the Sun from this book, and it fills a definite gap in the astronomical library of the general reader. The author worried me when he stated that in 1964 "the finding of the solar wind was a result of astronomical observations of extremely remote radio sources", but 86 pages later he changes his mind and Biermann's 1951-1953 observations of comet tail phenomena are given the credit. I also found that the position of the Sun in the stellar hierarchy needed much more emphasis and that it is not enough just to say that the Sun has "an average size, typical mass, normal structure, temperature and luminosity". Granted the Sun is on the Main Sequence but it is also about nine times more massive than the average star and about 2,000 times more luminous. But these are minor quibbles about a book which is generally of a high standard and is definitely a good read.

David $W$. Hughes is a Lecturer in Astronomy and Physics at the University of Sheffield.

\title{
The philosophy of astronomical discovery
}

\section{David S. Evans}

\section{Cosmic Discovery: The Search, Scope, and} Heritage of Astronomy. By Martin Harwit. Pp.334. ISBN UK 0-7108-0089-4; ISBN US 0-465-01428-3. (Harvester Press, Brighton/Basic Books, New York: 1981.) $£ 12.95, \$ 25$.

IN the blurb Sir Fred Hoyle describes Cosmic Discovery as "A remarkable book. A unique book". So it is. It is an examination of the epistemology of astronomy, and one of those books which will set astronomers and philosophers thinking and talking. It is not a book which will command general acquiescence. Exactly how much influence it will have is hard to say since it is certainly not everybody's cup of tea.

It starts off, oddly enough, with an idea connected with the collection of baseball cards. If one finds a duplicate, the set must be finite and if at any stage one counts up the number of single different cards $(A)$ and the number of duplicates $(B)$ the size of the set $(n)$ may be estimated from the statistical formula $n=A(A+2 B) / 2 B$. Harwit then applies this to astronomical discoveries of which he considers there are 43 distinct ones, and of these seven are recognized in two different ways. He is very careful about defining what he means by different ways, that is by totally unrelated 
techniques, perhaps best illustrated by the fact that the existence of galaxies was recognized by optical observations, but would now independently be found from radio observations. Putting $A=35, B=7$ in 1979 , with one triply recognized phenomenon $(C=1)$, he estimates that there are 123 separate cosmic phenomena to be found of which we have now recognized 35 per cent. From a discussion of the rate of discovery he thinks we might know all of them by $2150 \mathrm{AD}$, a somewhat depressing conclusion for those who love observational astronomy, though favourable error bars would increase the numbers and postpone the date. Harwit distinguishes between original discoveries and their development, so that our successors may not all be unemployed by then.

He builds upon a figure published by Halton Arp in 1965 which defines the limitations of observations of extended visual sources considered in terms of their absolute magnitudes and linear diameters. In an elaborate analysis of techniques he considers the parameters of possible detection methods - frequency, size, spectral resolution, time resolution, ellipticity of polarization and intensity - and constructs multidimensional phase diagrams to show what is now accessible and what might become accessible in the future, and classifies each of his fundamental discoveries according to this scheme.

One chapter outlines his selected set of phenomena and the circumstances of their discovery. He makes a number of points in passing - for example that new discoveries follow the introduction of new techniques, with which few readers will quarrel. However he tends to think only of revolutions in techniques rather than in their availability. To an observer one of the most important events of the past few decades was the identification by Thackeray and Wesselink of both Cepheids and RR Lyrae stars in the Magellanic Clouds because of the newly available 74-inch reflector in the Southern Hemisphere. The resultant doubling of the scale of the Universe does not rank in his list of 43 discoveries. He mentions the serendipity of many discoveries, but fails to mention the outstanding discovery by John B. Irwin that S Normae was a member of the cluster NGC 6087 . In fact his accounts of pulsating variables and flare stars leave a good deal to be desired and he fails to mention that pulsations continue down even into the white dwarf range. Indeed, one has the impression that in his accounts of fundamental discoveries Harwit confines himself to data from a rather restricted selection of well-known sources, especially those closest to the NASA publicity machine. For example, in his remarks on pulsars we fail to find any mention of the names of Cocke, Disney, Taylor, Nather or Warner, and the name of Lundmark is omitted from the story of the identification of the 1054 AD supernova remnant. The independent observation of the Uranus rings by Joseph Churms gets no mention. Among other distinguished absentees, de Vaucouleurs rates no inclusion for the discovery of the local supergalaxy.

Though one can quarrel with Harwit's selection of phenomena and his historical accounts, perhaps this will not invalidate his main thesis for some readers. He remarks that many of the discoverers were not originally trained as astronomers, which is true but easily explained. The basic discoveries in radio, X-ray or gamma-ray astronomy were made before anybody could describe himself as an astronomer specializing in these fields. Until a few decades ago, employment opportunities in astronomy were so few that even aspirant astronomers took care to have an employable skill in some other field as a hedge against failure in the discipline of first choice. He remarks that by his reckoning the major proportion of recent discoveries has been made by Americans, though he might have added that even now a high proportion of top posts in American astronomy are held by individuals whose original training was received elsewhere, apparently himself included.

Lastly, he notes that many of the most original discoveries have been made by individuals outside the main stream of astronomical organization and funding. This, of course, has been remarked in other sciences; one can command organized research but it seems impossible to command true originality. However, insofar as observational astronomers in all fields are now married to large and expensive instruments, detailed planning and allocation of resources is essential.

The last part of the book contains Harwit's message - "How should we organize astronomy?". He argues for 13 recommendations, many of which are obvious enough - better training in physics and mid-career training in new techniques for astronomers, and vigorous introduction of exponents of new techniques in astronomy. Long-term grants will better allow astronomers to address fundamental problems and the current peer review system must be loosened up to encourage true innovation, "which can seldom be justified in advance". On the other hand, projects should not be kept on just because they have gone on for a long time. He wishes to encourage gravitational wave and neutrino astronomy and believes that in the electromagnetic domain current observing capabilities could be increased by a factor of 1,000 at modest cost. He finishes with a plea for policy to be set by panels of generalists of "unusual breadth of interest and far-ranging vision", more frequent policy reviews and frequent analyses of the factors which contribute to astronomical success.

These are fine words, but how to implement them in the real world with limited funds for pure research is another matter. In view of Harwit's desire to have more frequent review committees, it is odd that he admits that "there is no evidence . . . that astronomical planning committees have in any way advanced the rate at which new astronomical phenomena are discovered ...". This is the paradox: astronomers need to spend a lot of money, much from public sources, and need to be accountable. They can guarantee the addition of large amounts of extremely valuable knowledge from their efforts. But the history of science demonstrates two things. One is that those who have predicted the exhaustion of the results of research have usually been proven wrong, however logical their arguments. The other is that discoveries of real originality are most often made by people who ignore the basic plan and pursue their own hunches. Many of them are rightly written off as cranks, but those who succeed are geniuses.

David S. Evans is a former Associate Director for Research at the McDonald Observatory and currently a Professor of Astronomy at the University of Texas at Austin.

\section{Relativity for all}

\section{D.J. Raine}

Discovering Relativity for Yourself. By Sam Lilley. Pp.425. ISBN hbk 0-521-23038-1; ISBN pbk 0-521-29780-X. (Cambridge University Press: 1981.) Hbk $£ 17.50, \$ 49.50$; pbk £7.95, \$19.95.

ONE approach to physical science for the intelligent but innumerate layman is through analogy and example, omitting the why and wherefore. Sam Lilley has a different approach. He believes that students can be taught how to work things out for themselves.

Schopenhauer opined that mathematical truths properly presented can, in any case, be grasped intuitively, thereby circumventing the aridity of the Euclidean mode, for which he gave as sole justification a pictogram proof of Pythagoras's theorem. Lilley has a beautiful extension of this to the invariance of the interval in special relativity. But, even when only elementary mathematics is involved, not everything can be presented so easily; for example, on p.74 we must digress from space-time diagrams to explain negative numbers.

With general relativity the difficulties multiply. The equivalence principle is

The latest edition of The Milky Way by Bart and Priscilla Bok has just been published by Harvard University Press. The book, which first appeared in 1941 and has now been revised for the fifth time, costs $£ 14$. 